\title{
Psychiatric Morbidities in Patients with Non-communicable Diseases among Inpatients of Medicine Department in a Tertiary Care Hospital: A Descriptive Cross-sectional Study
}

\author{
Kalpana Sharma, ${ }^{1}$ Govinda Dhungana, ${ }^{2}$ Shailendra Adhikari, ${ }^{3}$ Archana Bista Pandey, ${ }^{4}$ Muna Sharma ${ }^{5}$ \\ 'Department of Adult Health Nursing, Chitwan Medical College, Bharatpur, Chitwan, Nepal, ${ }^{2}$ Department of Statistics, \\ Birendra Multiple Campus, Bharatpur, Chitwan, Nepal, ${ }^{3}$ Department of Psychiatry, Chitwan Medical College, \\ Bharatpur, Chitwan, Nepal, ${ }^{4}$ Department of Women Health and Development, Institute of Medicine, Kathmandu, \\ Nepal, ${ }^{5}$ Department of Adult Health Nursing, Institute of Medicine, Kathmandu, Nepal.
}

\section{ABSTRACT}

Introduction: Psychiatric morbidities are common among patients with chronic non-communicable diseases. These diseases have high morbidity, mortality, and higher health costs. However, psychiatric conditions are often underdiagnosed and undertreated in our country. This study aimed to find out the psychiatric morbidities among patients with non-communicable diseases admitted in inpatients units of medicine department.

Methods: A descriptive cross-sectional study was conducted in inpatients of medicine department of a tertiary care hospital among 926 patients with chronic non-communicable diseases. Ethical approval was obtained from Chitwan Medical College Institutional Review Committee (Ref. No. CMC-IRC: 2074/75: 38). Convenient sampling technique was used. Patients were interviewed using the Patients Health Questionnaire. Data analysis was performed using Statistical Package for Social Sciences version 16.

Results: Among 926 non-communicable diseases patients, psychiatric morbidities observed were somatization $612(66.1 \%)$ anxiety 319 (34.4\%) and depression 379 (40.9\%). Patient with multiple non-communicable diseases had higher psychiatric morbidities compared to patients with a single disease.

Conclusions: Psychiatric morbidities are common among admitted patients suffering from noncommunicable diseases in Nepal. Hence, regular screening services are needed in all level of health care centres to identify and treat the risk groups on time.

Keywords: Nepal; non-communicable diseases; patients; psychiatric morbidities.

\section{INTRODUCTION}

Non-communicable diseases (NCDs) are the leading cause of deaths globally. ${ }^{1}$ It accounts for more than $85 \%$ of premature deaths occurred in low and middleincome countries. ${ }^{2}$ Psychiatric morbidities such as depression and anxiety are high in NCDs patients. ${ }^{3,4}$ They are collectively responsible for $72 \%$ of all deaths. ${ }^{5}$ The co-existence of psychiatric disorders with NCDs has important implications on quality of life, general well-being, cost of treatment and general longevity of the patient. ${ }^{3,6}$
In Nepal, NCDs accounts for $66 \%$ of deaths in $2016 .^{7}$ The prevalence of mental disorders is higher among physically ill patients $(31.7 \%)^{8}$ compared to healthy adults (13.2\%). ${ }^{9}$ These conditions are underdiagnosed and undertreated in our country due to social stigma. Also, there is limited literature on the prevalence of mental disorders among patients with NCDs.

Correspondence: Dr. Kalpana Sharma, Chitwan Medical College, Bharatpur, Nepal. Email: sharma.kalpana@cmc.edu.np, Phone: +977-9845023118. 
This study aimed to find out psychiatric morbidities among NCDs patients admitted in inpatients units of medicine department of Chitwan Medical College.

\section{METHODS}

A descriptive cross-sectional study was conducted in inpatients of medicine department of Chitwan Medical College Teaching Hospital, Chitwan from 15th June 2018 AD to 17th September 2019 AD. Ethical approval was obtained from the Chitwan Medical College Institutional Review Committee (Ref. No. CMC-IRC: 2074/75: 38). The study population was those patients who were clinically diagnosed to have NCDs such as type-Il diabetes mellitus (DM), hypertension (HTN), coronary artery diseases (CAD), chronic kidney disease (CKD) and chronic obstructive pulmonary diseases (COPD) either alone or in combination for 1 year and above and were admitted in different inpatients units of medicine department.

Sample size calculation,

$\mathrm{n}=\mathrm{Z}^{2} \times \mathrm{p} \times(1-\mathrm{p}) / \mathrm{e}^{2}$

$=(1.96)^{2} \times 0.317 \times 0.683 /(0.03)^{2}$

$=925$

Where,

$\mathrm{n}=$ Sample size

$Z=1.96$ at $95 \%$ Confidence Interval

$\mathrm{p}=$ estimated prevalence rate $(31.7 \%)=0.317$

$\mathrm{e}=$ Margin of error, $3 \%$

The sample size of 925 patients was calculated considering the confidence level of $95 \%$. Convenient sampling technique was used for recruiting the desired number of samples in the study. Structured interview schedule for the socio-demographic and diseaserelated information and Patient Health Questionnaire (PHQ) for the screening of psychiatric morbidities were used for the data collection. PHO contains 3 parts: PHQ-15 to assess somatic functions, Generalized Anxiety Disorders-7 (GAD-7) to assess anxiety and $\mathrm{PHQ}-9$ to assess depression. The $\mathrm{PHO}$ questionnaire is a validated questionnaire, which found to be useful in the screening of patients for psychiatric illness

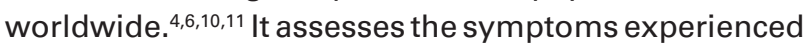
by participants during the 2 weeks before they take the survey. Each item of GAD-7 and PHQ-9 was rated 0 to 3 scores where 0-not at all, 1-several days, 2-more than half of the days, and 3-nearly every day, with higher scores indicating patients' increased self-report of anxiety and depression severity. Scores obtained in GAD-7 was classified into mild (5-9), moderate $(10-14)$, and severe anxiety $(\geq 15)$. Likewise, scores of PHO-9 was divided into mild $(5-<10)$, moderate (10$<15)$, moderately severe $(15-<20)$ and severe $(\geq 20)$ depression.

To maintain the rights of patients, further evaluation was done for those patients who were found to be positive on $\mathrm{PHO}$ by psychiatrists using Standard Beck Anxiety Scale ${ }^{12}$ and Hamilton Depression Scale ${ }^{13}$ for the confirmation and further treatment. Written informed consent was obtained from the patients ensuring their confidentiality of the information. Collected data were entered into Epi data 3.1 and exported to IBM SPSS version 20. Then data were analyzed in terms of descriptive statistics such as frequency, and percentages.

\section{RESULTS}

A total of 926 patients participated in the study. Among them, $548(59.2 \%)$ were female and the mean age was $61.12( \pm 17.79)$ years. More than two-thirds of the patients were residing in the urban area $624(67.4 \%)$, married $677(73.2 \%)$ and belonged to the joint family $682(73.7 \%)$. Nearly half of the patients were illiterate $461(49.8 \%)$, involved in agriculture 453 (48.9\%) and reported that their family income was just sufficient for the daily expenses. However, more than twothird $627(67.7 \%)$ of the patients reported the illness impact on their job as a result they quitted their job. Regarding the behavioural pattern, nearly half 451 $(48.7 \%)$ of the patients were never smoker, and 122 $(13.2 \%)$ of patients were still smoking a cigarette. Only $203(21.9 \%)$ of patients had a habit of alcohol use. Regarding clinical variables, more than one-quarter of patients had multiple NCDs 356 (38.4\%) followed by COPD 188 (20.3\%), diabetes mellitus 110 (11.9\%) and HTN 102 (11.0\%). More than half 535 (57.8\%) of the patients' duration of diagnosis was less than 5 years and $532(64.3 \%)$ of patients had a history of hospital admission in the last year (Table 1).

\begin{tabular}{|c|c|c|c|}
\hline Variables & n (\%) & Variables & n (\%) \\
\hline Age groups in years & & Occupation & \\
\hline$<30$ & $31(3.3)$ & Agriculture & $453(48.9)$ \\
\hline $30-49$ & $148(16.1)$ & Homemade work & $283(30.6)$ \\
\hline $50-69$ & 444 (47.9) & Earn Cash & $141(15.2)$ \\
\hline $70+$ & $303(32.7)$ & Others & $49(5.3)$ \\
\hline
\end{tabular}


Sharma et al. Psychiatric Morbidities in Patients with Non-communicable Diseases among Inpatients of Medicine Department in a Tertiary...

\begin{tabular}{|c|c|c|c|}
\hline $\begin{array}{l}\text { Variables } \\
\text { Mean } \pm S D=61.12 \pm 17.79\end{array}$ & $\mathrm{n}(\%)$ & $\begin{array}{l}\text { Variables } \\
\text { Illness impact on job }\end{array}$ & n (\%) \\
\hline Sex & & Yes & $627(67.7)$ \\
\hline Female & $548(59.2)$ & No & $299(32.3)$ \\
\hline Male & $378(40.8)$ & Family income & \\
\hline Religion & & Insufficient for daily expenses & $229(24.7)$ \\
\hline Hindu & $787(85.0)$ & Just sufficient for daily expenses & $410(44.3)$ \\
\hline Others(C) & $139(15.0)$ & Surplus for daily expenses & $287(31.0)$ \\
\hline Caste & & Food habit & \\
\hline Bramin & $396(39.8)$ & Vegetarian & $195(21.1)$ \\
\hline Chhetri & $152(16.4)$ & Non-vegetarian & 731 (78.9) \\
\hline Janajati & $308(33.3)$ & Smoking habit & \\
\hline Dalit and Madhesi & $97(10.5)$ & Smoker & $122(13.2)$ \\
\hline Place of residence & & Past smoker & $353(38.1)$ \\
\hline Rural & $302(32.6)$ & Never smoker & $451(48.7)$ \\
\hline Urban & $624(67.4)$ & Alcohol intake & \\
\hline Marital status & & Yes & 203 (21.9) \\
\hline Unmarried & $27(2.9)$ & No & $723(78.1)$ \\
\hline Married & $677(73.2)$ & Type of NCDs & \\
\hline Widow /widower & $218(23.5)$ & Diabetes mellitus & $110(11.9)$ \\
\hline Divorce and others & $4(0.4)$ & Hypertension & $102(11.0)$ \\
\hline Family type & & Coronary artery disease & $97(10.5)$ \\
\hline Nuclear & $244(26.3)$ & Chronic kidney disease & $73(7.9)$ \\
\hline Joint & $682(73.7)$ & Chronic obstructive pulmonary diseases & $188(20.3)$ \\
\hline Current living status & & Multiple NCDs & $356(38.4)$ \\
\hline Alone & $98(10.6)$ & $\begin{array}{l}\text { Duration of disease diagnosis in the } \\
\text { years }\end{array}$ & \\
\hline With family & $828(89.4)$ & $<5$ & $535(57.8)$ \\
\hline Education status & & $\geq 5$ & $391(42.2)$ \\
\hline Illiterate & $461(49.8)$ & Hospital admission in the last one year & \\
\hline Literate & $266(28.7)$ & None & $331(35.7)$ \\
\hline Primary (1-8) & $107(11.6)$ & $<3$ & 481 (51.9) \\
\hline Secondary and above & $92(9.9)$ & $\geq 3$ & $114(12.3)$ \\
\hline
\end{tabular}

(C) Others: Buddish, Cristian Muslim, Kirat and others direct cash

६Earn Cash: business, service and daily wage who earn

Out of 926 patients with NCDs, symptoms of somatization, depression and anxiety were observed among 612 $(66.1 \%), 379(40.9 \%)$ and $319(34.4 \%)$ respectively. The higher proportion of $679(73.3 \%)$ of patients with multiple NCDs showed evidence of psychiatric morbidities compared to patients with single NCDs. Similarly, the prevalence of psychiatric morbidities was proportionally higher among patients with DM, HTN, and CKD than patients with other NCDs (Table 2).

\begin{tabular}{|llll|}
\hline \multicolumn{4}{|l|}{ Table 2. Psychiatric morbidities among patients with NCDs. } \\
\hline Types of NCDs & Somatization $\mathrm{n}(\%)$ & Anxiety $\mathrm{n}(\%)$ & Depression $\mathrm{n}(\%)$ \\
Diabetes Mellitus $(\mathrm{n}=110)$ & $81(73.6)$ & $48(43.6)$ & $55(50.0)$ \\
Hypertension $(\mathrm{n}=102)$ & $73(71.6)$ & $29(28.4)$ & $32(31.4)$ \\
Coronary artery disease $(\mathrm{n}=97)$ & $34(35.1)$ & $15(15.5)$ & $15(15.5)$ \\
Chronic kidney disease $(\mathrm{n}=73)$ & $49(67.1)$ & $29(39.7)$ & $36(49.3)$ \\
Chronic obstructive pulmonary disease $(\mathrm{n}=188)$ & $89(47.3)$ & $20(10.6)$ & $26(13.8)$ \\
Multiple NCDs $(\mathrm{n}=356)$ & $286(80.3)$ & $178(50.0)$ & $215(60.4)$ \\
Total $(\mathrm{n}=926)$ & $612(66.1)$ & $319(34.4)$ & $379(40.9)$ \\
\hline
\end{tabular}

Overall, the symptoms of somatization were found among $612(66.1 \%)$ of NCDs patients where mild, moderate and severe symptoms of somatization were 360 (38.9\%), $198(21.4 \%), 54(5.8 \%)$ respectively (Table 3). 
Sharma et al. Psychiatric Morbidities in Patients with Non-communicable Diseases among Inpatients of Medicine Department in a Tertiary...

\begin{tabular}{|c|c|c|c|c|c|}
\hline \multirow[t]{2}{*}{ Types of NCDs } & \multicolumn{5}{|c|}{ Level of Somatization } \\
\hline & None $\mathrm{n}(\%)$ & Mild n (\%) & Moderate $\mathrm{n}(\%)$ & Severe $\mathrm{n}(\%)$ & Total n (\%) \\
\hline Diabetes Mellitus ( $n=110)$ & $29(26.4)$ & $34(30.9)$ & $39(35.5)$ & $8(7.3)$ & $81(73.6)$ \\
\hline Hypertension $(n=102)$ & $29(28.4)$ & $47(46.1)$ & $19(18.6)$ & $7(6.9)$ & $73(71.6)$ \\
\hline Coronary artery disease $(n=97)$ & $63(64.9)$ & $26(26.8)$ & $8(8.2)$ & $0(0.0)$ & $34(35.1)$ \\
\hline Chronic kidney disease $(n=73)$ & $24(32.9)$ & $30(41.1)$ & $19(26.0)$ & $0(0.0)$ & $49(67.1)$ \\
\hline $\begin{array}{l}\text { Chronic obstructive pulmonary } \\
\text { disease }(n=188)\end{array}$ & $99(52.7)$ & 75 (39.9) & $8(4.3)$ & $6(3.2)$ & $89(47.3)$ \\
\hline Multiple NCDs ( $n=356)$ & 70 (19.7) & $148(41.6)$ & $105(29.5)$ & $33(9.3)$ & $286(80.3)$ \\
\hline Total $(n=926)$ & $314(33.9)$ & $360(38.9)$ & $198(21.4)$ & $54(5.8)$ & $612(66.1)$ \\
\hline
\end{tabular}

The symptoms of anxiety were proportionally higher among patients with multiple NCDs 178 (50.0\%). Among single NCDs, the symptoms of anxiety were proportionally higher in patients with DM 48 (43.6\%) and CKD 29 $(39.7 \%)$ as compared to other diseases. Overall, 319 (34.4\%) of patients with NCDs had anxiety symptoms where $164(17.7 \%), 128(13.8 \%)$, and $27(2.9 \%)$ had mild, moderate and severe depression respectively (Table 4).

\begin{tabular}{|c|c|c|c|c|c|}
\hline \multirow[t]{2}{*}{ Types of NCDs } & \multicolumn{5}{|c|}{ Level of Anxiety } \\
\hline & None $\mathrm{n}(\%)$ & Mild n (\%) & Moderate n (\%) & Severe $\mathrm{n}(\%)$ & Total n (\%) \\
\hline Diabetes mellitus ( $n=110)$ & $62(56.4)$ & $20(18.2)$ & $26(23.6)$ & $2(1.8)$ & $48(43.6)$ \\
\hline Hypertension ( $n=102)$ & $73(71.6)$ & $21(20.6)$ & $3(2.9)$ & $5(4.9)$ & $29(28.4)$ \\
\hline Coronary artery disease $(n=97)$ & $82(84.5)$ & $3(3.1)$ & $12(12.4)$ & 0 & $15(15.5)$ \\
\hline Chronic kidney disease $(n=73)$ & $44(60.3)$ & $15(20.5)$ & $14(19.2)$ & 0 & $29(39.7)$ \\
\hline $\begin{array}{l}\text { Chronic obstructive pulmonary } \\
\text { disease }(n=188)\end{array}$ & $168(89.4)$ & $14(7.4)$ & $6(3.2)$ & 0 & $20(10.6)$ \\
\hline Multiple NCDs ( $n=356)$ & $178(50.0)$ & $91(25.6)$ & $67(18.8)$ & $20(5.6)$ & $178(50.0)$ \\
\hline Total $(n=926)$ & $607(65.6)$ & $164(17.7)$ & $128(13.8)$ & $27(2.9)$ & $319(34.4)$ \\
\hline
\end{tabular}

The evidence of depressive symptoms was higher in patients with multiple NCDs that is, 215 (60.4\%) followed by diabetes 55 (50.0\%) and CKD 36 (49.3\%). Overall, 379 (40.9\%) of NCDs patients had depressive symptoms where mild, moderate and severe depressions among patients with NCDs were 196 (21.2\%), 167 (18.1\%), and 16 (1.7\%) respectively (Table 5).

\begin{tabular}{|llllll|}
\hline \multicolumn{7}{|l|}{ Table 5. Level of depression among patients with NCDs $(\mathrm{n}=926)$. } \\
\hline
\end{tabular}

DISCUSSION

Our study revealed that the psychiatric morbidities are common among the patients suffering from chronic NCDs such as DM, HTN, CAD, COPD and CKD. Two in three NCDs patients suffer from the symptoms of somatization whereas one in three patients have anxiety and depressive symptoms. A higher proportion of patients with multiple NCDs showed the evidence of symptoms of somatization, anxiety and depression compared to patients with single NCDs. 
Prevalence of somatization symptoms was found to be $66.1 \%$ among the patients with NCDs which was higher than the available evidence ${ }^{6}$ where the prevalence of somatization was $35.1 \%$. These differences in studies finding might be due to different criteria of sample selection and study settings. While observing the level of somatization among patients, $38.9 \%$ had mild, $21.4 \%$ had moderate and $5.8 \%$ had severe somatic symptoms.

The prevalence of anxiety symptoms was found to be $34.4 \%$ among our study participants in which $17.7 \%$; $13.8 \%$ and $2.9 \%$ of patients respectively had mild, moderate and severe anxiety disorders. This finding is slightly higher than the finding of the study conducted in India6in which $19.1 \%$ of patients had anxiety symptoms whereas our findings are almost similar with the study conducted in another study in India done by Verma et al. which showed $38.7 \%$ prevalence of anxiety among the cases. ${ }^{14}$ However, Taneja et al. showed a higher prevalence of depression (i.e. 56.7\%) among NCDs cases. ${ }^{4}$ These studies finding might be varied due to the use of different data collection tool, characteristics of patients and data collection settings which might influence the prevalence of anxiety among patients.

In this study, the prevalence of depressive symptoms among NCDs patients was $41.0 \%$ which was found to be higher than the study done by Rijal et al. in 2016 in the general population of Nepal which showed $11.7 \%$ prevalence. ${ }^{15}$ It indicates that depression is higher among NCDs cases. However, available evidence showed varied results regarding the prevalence of depression among NCDs cases. The prevalence observed in our study is higher compared to other study done in India in which $29.1 \%$ of patients had depressive symptoms ${ }^{6}$ whereas lower than the study conducted in the urban slum of East Delhi which found depression among more than half $(56.4 \%)$ of NCDs patients. ${ }^{4}$ The difference in findings might be due to difference in characteristics of samples included in the study. In our study included five types of NCDs patients whereas these two studies excluded patients with COPD and CKD in their studies. ${ }^{4,6}$ However, our observation in general are consistent with the prevalence of depression reported by Verma et al. which showed $58.1 \%$ of depression among NCDs patients. ${ }^{14}$ On the severity of depression, our study revealed $21.2 \%$ mild, $18.1 \%$ moderate and $1.7 \%$ severe depression among the patients.

Our country is also giving priorities for noncommunicable diseases and mental health through implementing sustainable developmental goal in national health policy. Nepal health research council, university grant commission and other NGOs have also mentioned NCDs and mental health as a priorities area for funding. Exploring psychiatric morbidities in patients with major non-communicable diseases using standard screening tool is the strong point of this study. Despite this, our study has certain limitations like- it was conducted in only one study setting and hospital admitted patients only, which may limit the generalizability of the study and it did not exclude the patients with other health problems which might have influenced the study findings.

\section{CONCLUSIONS}

Psychiatric morbidities i.e. anxiety, depression and symptoms of somatization, is common among the patients suffering from non-communicable diseases such as diabetes, hypertension, chronic obstructive pulmonary disease, cardiovascular diseases and chronic kidney disease in Nepal. Anxiety and depression are proportionally higher in patients with DM and CKD than other NCDs patients. Likewise, patient with multiple NCDs tends to have higher psychiatric morbidities compared to patients with a single disease. Hence, there is a need to develop an integrated care model to manage these morbidities to the vulnerable groups at all level.

\section{ACKNOWLEDGEMENTS}

Authors would like to express sincere thanks to the management of Chitwan Medical College, department of medicine and psychiatry for their kind co-operation during the data collection period. We are also grateful to the University Grant Commission for their financial support. Last but not the least researchers are thankful to all participants, without their kind support and participation this study was not possible.

\section{Conflict of Interest: None.}

\section{REFERENCES}

1. World Health organization (WHO). Non-communicable diseases. 2018. [Full Text]

2. WHO. Global status report on non-communicable diseases Geneva, Switzerland. 2014. [Full Text]

3. Mukeshimana M, Mchunu G. The co-morbidity of depression and other chronic non-communicable diseases: a review of literature on the epidemiology, diagnosis and health effects. Rwanda Journal. 2016;3(1):44-50. [Full Text | DOI]

4. Taneja N, Adhikary M, Chandramouleeswaan S, Kapoor SK. Prevalence of common mental disorders among patients with diabetes mellitus and hypertension in an Urban East Delhi Slum - A cross sectional study. TJP. 2015;1(1):27-31. [Full Text] 
5. WHO. Non communicable diseases and mental health. 2020. [Full Text]

6. Kulkarni V, Chinnakali P, Kanchan T, Rao A, Shenoy M, et al. Psychiatric co-morbidities among patients with select non-communicable diseases in a Coastal City of South. India Int J Prev Med. 2014;5(9):1139-45. [라bMed]

7. Nepal Health Research Council (NHRC), Ministry of Health and Population (MoHP) and Monitoring Evaluation and Operation Research (MEOR). Nepal Burden of Diseases 2017: A Country Report based on the Global Burden of Disease 2017 study. Kathmandu, Nepal: NHRC, MoHP, MEOR;2019. [Full Text]

8. Shyangwa P, Joshi D, Sherchan S, Thapa K. Psychiatric morbidity among physically ill persons in eastern Nepal. Nepal Medical College Journal. 2009;11(2):118-22. [․ㅏll Text]

9. Jha AK, Ojha SP, Dahal S, Sharma P, Pant SB, Labh S, et al. Prevalence of mental disorders in Nepal: Findings from the pilot study. JNHRC. 2019;17(43):141-7. [Full Text | DOI ]

10. Barua A,Jacob, GP, MahmoodSS. Patienthealth questionnaire for screening psychiatric disorders in secondary healthcare. Indian J Psychiatry. 2013;55(2). [PubMed | DOI]
11. Kroenke K, Spitzer RL, Williams JBW, Lowe B. The patient health questionnaire somatic, anxiety, and depressive symptom scales: A systematic review. General Hospital Psychiatry. 2010;32,345-59. [Full Text | DOI ]

12. Beck AT, Epstein N, Brown G, Steer RA. Beck Anxiety inventory for measuring clinical anxiety. Journal of Consulting and Clinical Psychology. 1988;56,893-7. [․ull Text]

13. Hamilton, M. The Hemilton Rating Scale for Depression. Journal of Occupational Psychiatry. 1979;10(2),149-65. [․ull Text]

14. Verma M, Grover S, Tripathy JP, Singh T, Nagaraja SB, Kathirvel S, Singh G, and Nehra R. Co-existing non-Communicable diseases and mental illnesses amongst the elderly in Punjab, India. European Endocrinology. 2019;15(2):106-12. [PubMed]

15. Risal A, Manandhar K, Linde M, Steiner TJ, Holen A. Anxiety and depression in Nepal: Prevalence, comorbidity and associations'. BMC Psychiatry. 2016;16:102. [PubMed | $\underline{\mathrm{DOI}}]$ 